\title{
A theoretical study of the electronic spectrum of bithiophene
}

\author{
Mercedes Rubio, Manuela Merchán, and Enrique Ortí \\ Departamento de Química Física, Universitat de València, Dr. Moliner 50, Burjassot, \\ E-46100 Valencia, Spain \\ Björn O. Roos \\ Department of Theoretical Chemistry, Chemical Centre, P.O.B. 124, S-221 00 Lund, Sweden
}

(Received 12 October 1994; accepted 23 November 1994)

\begin{abstract}
The electronic spectrum of bithiophene in the energy range up to $6.0 \mathrm{eV}$ has been studied using multiconfigurational second order perturbation theory (CASPT2) and a basis set of ANO type, with split valence quality and including polarization functions on all heavy atoms. Calculations were performed at a planar (trans) and twisted geometry. The calculated ordering of the excited singlet states is ${ }^{1} B_{u},{ }^{1} B_{u},{ }^{1} A_{g},{ }^{1} A_{g}$, and ${ }^{1} B_{u}$ with $0-0$ transition energies: $3.88,4.15,4.40,4.71$, and 5.53 $\mathrm{eV}$, respectively. The first Rydberg transition $(3 s)$ has been found at $5.27 \mathrm{eV}$. The results have been used in aiding the interpretation of the experimental spectra, and in cases where a direct comparison is possible there is agreement between theory and experiment. (C) 1995 American Institute of Physics.
\end{abstract}

\section{INTRODUCTION}

The 2,2'-bithiophene molecule has been studied extensively, since it is the smallest building block (except thiophene itself) of polythiophene. ${ }^{1-9}$ The idea is that studies of the small oligomers will give the key to the understanding of the electronic structure of polythiophene. Working with small oligomers avoids problems such as those found in the synthesis of the polymer: the resulting polymer usually depends on the procedure employed for the synthesis, and consequently the spectroscopic experiments, as well as the physical properties of the material, are sensitive to the sample preparation. ${ }^{10}$ In theoretical studies it is possible to use higher level methods in studies of small oligomers, and extrapolation of computed properties to the polymer might give results more reliable than those obtained with the polymer itself.

The lowest excited states, in addition to the geometry of the molecule, have been the most important items investigated. There is a great interest in determining the symmetry of the lowest excited state $(A$ or $B)$ and in knowing if the order of the lowest excited states is equal or different from that found in polyenes. Due to their structural simplicity, linear polyenes and polyacetylene have been the focus of most of the experimental and theoretical studies. It is experimentally known that for the longer all-trans polyenes, starting with hexatriene, ${ }^{11}$ the lowest singlet $0-0$ transition corresponds to an electronic state of the same symmetry as the ground state (for example, in dodecahexaene the $2{ }^{1} A_{g}$ state is $0.6 \mathrm{eV}$ below the $1{ }^{1} B_{u}$ state). Experimental results for bithiophene and $\alpha$-hexathiophene show, on the other hand, that in both molecules the $2{ }^{1} A_{g}$ state is higher in energy than the $1^{1} B_{u}$ state (0-0 transition). ${ }^{1,2,12,13}$ There have been no direct measurements of the location of the $2{ }^{1} A$ state in polythiophene, and therefore, all the predictions about the ordering of the $2{ }^{1} A$ and $1{ }^{1} B$ states in the polymer are hypothetical. Thus, both possibilities have been suggested. Birnbaum and Kohler $^{2}$ consider it highly probable that the order of the $2^{1} A$ and $1{ }^{1} B$ states reverses for polythiophenes containing more than six thiophene rings. They have observed the $0-0$ band of the transition to the $2{ }^{1} A$ state $0.81 \mathrm{eV}$ above the $0-0$ band of the $1^{1} A \rightarrow 1{ }^{1} B$ transition in bithiophene. ${ }^{2}$ The fact that the energy difference between these two $0-0$ bands is much smaller in hexathiophene $(0.11 \mathrm{eV})$, although the same ordering is found, suggests that the reversed order prevails for longer chains ( $>6$ monomer units), that is, $2{ }^{1} A$ is below $1{ }^{1} B$. Periasamy et al. ${ }^{12,13}$ have, however, suggested that the electronic energetics of "real" polythiophene follows the same excited states ordering as in hexathiophene based on the similarity observed between the photoluminescence of the polymer and the oligomer.

Theoretical studies of the lowest excited states of thiophene oligomers have been recently reported. ${ }^{7-9}$ Beljonne et $a l .{ }^{7}$ have analyzed the evolution of the state ordering as a function of chain length. They have calculated the lowest singlet excited states for thiophene oligomers containing from one up eight rings using the semiempirical INDO/CI method and AM1 optimized geometries. The geometries of the excited states $1{ }^{1} B_{u}$ and $2{ }^{1} A_{g}$ were optimized using two different approaches. They predict the two lowest excited states of bithiophene to be in reversed order with respect to experiment. ${ }^{1,2}$ By a modification of the CI expansion they managed to reproduce the experimental order.

The two lowest excited states of thiophene oligomers from 1 up 4 rings have been extensively studied by Negri and Zgierski. ${ }^{8}$ Semiempirical methods were used to compute the excitation energies and oscillator strengths. Different approaches, including $a b$ initio methods, have been employed to optimize the geometries of the ground and excited states. Although the vertical excitation energies place the $2{ }^{1} A_{g}$ state of bithiophene above the $1{ }^{1} B_{u}$ state, the reversed order is predicted for the $0-0$ transitions $(3.8 \mathrm{eV}$ for the $0-0$ transition to the $2{ }^{1} A_{g}$ state and 4.1 for the $0-0$ transition to the $1{ }^{1} B_{u}$ state). Based on these results, Negri and Zgierski ${ }^{8}$ claim that the two lowest excited states of thiophene oligomers and polyenes are similar, and therefore the lowest excited state is predicted to have the same symmetry as the ground state, which is contrary to the experimental 
TABLE I. Influence of the basis set on the valence excitation energies and oscillator strengths of thiophene computed at the CASPT2 level.

\begin{tabular}{cccccc}
\hline \hline & \multicolumn{2}{c}{ TW $^{\mathrm{a}}$} & & \multicolumn{2}{c}{ Previous $^{\mathrm{b}}$} \\
\cline { 2 - 3 } \cline { 5 - 6 } State & $\Delta E$ & Osc. Str. & & $\Delta E$ & Osc. Str. \\
\hline$V_{1}:{ }^{1} A_{1}$ & 5.15 & 0.084 & & 5.33 & 0.089 \\
$V_{2}:{ }^{1} B_{2}$ & 5.58 & 0.087 & & 5.72 & 0.070 \\
$V_{3}:{ }^{1} A_{1}$ & 6.79 & 0.338 & & 6.69 & 0.185 \\
$V_{4}:{ }^{1} B_{2}$ & 7.29 & 0.427 & & 7.32 & 0.392 \\
\hline \hline
\end{tabular}

${ }^{a}$ Basis set: $C[3 s 2 p 1 d] / S[4 s 3 p 1 d] / H[2 s]$.

basis set: $C[4 s 3 p 1 d] / S[5 s 4 p 2 d] / H[2 s 1 p]+1 s 1 p 1 d$ diffuse functions (Ref. 15).

evidence. ${ }^{1,2,12,13}$ In order to explain the experimental information, they suggest that the ${ }^{1} A_{g}$ experimentally observed is the $3{ }^{1} A_{g}$ state. Here, one could note that a comparison of the two lowest excited states of 1,3 cis-butadiene and thiophene shows important differences. High level $a b$ initio calculations find the first excited state in thiophene to be of the same symmetry as the ground state $\left(A_{1}\right)$. This is not the case in cis-butadiene, where instead the excited state symmetry is $B_{1}$. The oscillator strengths have the same order of magnitude in the two lowest excited states of thiophene in contrast to the values reported for butadiene. ${ }^{14-16}$ In addition, the multiconfigurational wave function of the $2{ }^{1} A_{1}$ state is different in the two molecules, having a larger contribution of double excitations in 1,3 cis-butadiene (50\% vs $16 \%$ ). We disagree therefore with the similarity proposed by Negri and Zgierski ${ }^{8}$ between thiophene oligomers and polyenes. A simple examination of the molecular orbitals of bithiophene shows the important contribution of the $S$ atom.

Here, we shall present results from a high level ab initio study of the lower excited states of bithiophene. The complete active space SCF method has been used in combination with multiconfigurational second order perturbation theory and an adequate basis set which includes polarization functions on all centers. We shall show that the electronic spectrum cannot be simply related to that of the small polyenes, or even to thiophene itself, since the coupling between the two units is strong and the molecular orbitals do not retain the characteristics of the monomer orbitals. The computed spectrum will be shown to be in full agreement with the most recent experimental information. A detailed discussion of the experimental situation will be given in the result section.

\section{COMPUTATIONAL DETAILS}

Generally contracted basis sets of atomic natural orbital (ANO) type are used, which have been obtained from $\mathrm{S}(17 s 12 p 5 d) / \mathrm{C}(14 s, 9 p, 4 d) / \mathrm{H}(8 s)$ primitive sets. ${ }^{17,18}$ The contraction scheme used in most calculations was $\mathrm{S}[4 s 3 p 1 d] / \mathrm{C}[3 s 2 p 1 d] / \mathrm{H}[2 s]$. Table I shows the results of control calculations performed for the valence excited states of thiophene. For sake of comparison, the results obtained in a comprehensive study of the electronic spectrum of thiophene, using a more flexible valence contraction scheme and diffuse functions, are also included. ${ }^{15}$ The present basis set is able to describe the valence excited states within the required accuracy and therefore can be confidently employed in the study of larger and related systems like bithiophene. In addition, in order to locate the lowest Rydberg state, the original basis set was supplemented with two $s$ - (exponents 0.002666 and 0.007235 ) and two $p$-type (exponents 0.002332 and 0.006331 ) diffuse functions placed at the charge centroid of the molecule. This enlarged basis set was, however, only used in the calculation of the Rydberg state.

Calculations have been performed at the planar trans geometry $\left(C_{2 h}\right.$ symmetry $)$ and at the twisted transbithiophene conformation $\left(C_{2}\right.$ symmetry) obtained at the MP2/6-31G* level. ${ }^{19}$ At the same level of calculation, the twist angle between the two rings was computed to be $38^{\circ}$ at the equilibrium geometry, with a small barrier to transplanarity $(\approx 1 \mathrm{kcal} / \mathrm{mol})$. Electron diffraction experiments have determined that bithiophene is twisted in the gas phase. ${ }^{20,21}$ The most recent experiment ${ }^{21}$ has shown the existence of two conformations. The planar trans geometry has been only observed in crystals. ${ }^{22}$ As will be discussed below, the reduction of symmetry upon rotation around the central $\mathrm{C}-\mathrm{C}$ bond plays a key role in the interpretation of the spectrum. The molecule was placed in the $x y$ plane, with the long molecular axis corresponding to the $y$ axis. The $\pi$ orbitals belong therefore to the $b_{g}$ and $a_{u}$ irreducible representations of the $C_{2 h}$ symmetry group.

Multiconfigurational wave functions were initially determined at the complete active space (CAS)SCF level of approximation. ${ }^{23}$ In most of the calculations the active space comprises the ten valence $\pi$ orbitals. It may happen, however, that large coefficients appear in the first order wave function due to the appearance of, so-called, intruder states in the first order interacting space. If this happens, the active space has to be enlarged in order to include these states into the CAS CI space and thus make them noninteracting with the reference function. Such a situation occurred in the present study for the $2{ }^{1} A_{g}$ excited states in the twisted conformation. Two orbitals (one of each symmetry) had to be added. The orbitals were of diffuse character and only weakly interacting with the remaining configurations. The effect on the excitation energies is therefore small. Still, this single excitation energy may carry a larger uncertainty than the remaining ones, since it was computed with a different active space. The $12 \pi$ electrons were active. All remaining valence electrons were inactive, including the sulphur $2 s$ and $2 p$ electrons. The carbon and sulphur $1 s$ electrons were kept frozen in the form determined by the ground state SCF wave functions and were not correlated. The relative energy of each excited state refers to the ground state energy computed with the same active space. The molecular orbitals (MOs) for the excited states have been obtained from average CASSCF calculations, where the averaging includes all states of interest for a given symmetry.

The CASSCF wave functions are used as reference functions in the second order perturbation treatment through the CASPT2 method. ${ }^{24,25}$ Dynamic correlation effects, which are important for accurate predictions of the excitation energies, are accounted for in this way. The performance of the method in calculations of electronic spectra has been discussed in detail in earlier applications. ${ }^{26}$

The dipole transition moments are computed using the 
CASSCF state interaction (CASSI) method. ${ }^{27}$ Energy differences corrected by CASPT2 are used in the oscillator strength formula.

The calculations have been performed on IBM RS/6000 workstations of the University of Valencia using the MOLCAS-3 quantum chemistry software. ${ }^{28}$

\section{RESULTS}

In this section we shall discuss the results of the calculations. In order to give background for this discussion we first summarize the experimental situation. Before discussing the computed energies and molecular properties, a simple qualitative analysis, based on orbital energy differences, will be made that shows why we can expect to find a number of electronic states with low energies.

\section{A. Experimental background}

Kohler et al. have recently reported the fluorescence excitation and fluorescence spectra of bithiophene seeded in a supersonic helium expansion ${ }^{4}$ and in solid solutions in hexane at $4.2 \mathrm{~K}$. ${ }^{1}$ The vibronic progressions in both the fluorescence excitation spectra are very similar, which suggest that the molecular conformation of bithiophene is similar in the gas and the condensed phases. A solvent to gas shift of 1508 $\mathrm{cm}^{-1}(0.19 \mathrm{eV})$ is observed. ${ }^{4}$ Changing the expansion conditions, the fluorescence excitation spectra of the cis and trans isomers can be separated. The $S_{1}$ origin excitation energy was located at $31100 \mathrm{~cm}^{-1}(3.86 \mathrm{eV})$ for the trans isomer ${ }^{4}$ in a supersonic He expansion, and at $29600 \mathrm{~cm}^{-1}$ $(3.67 \mathrm{eV})$ in condensed phase. ${ }^{1}$ Only the spectrum of the trans conformer was observed in the condensed phase experiments. Birnbaum and Kohler ${ }^{1}$ assign the $S_{1}$ state to the $1{ }^{1} B_{u}$ state, which they assume to be described by the $\mathrm{HOMO} \rightarrow$ LUMO configuration. The overlap of the $0-0$ band in the excitation and emission spectra, as well as the similar vibrational development, indicate that the $S_{0} \rightarrow S_{1}$ transition is symmetry allowed, supporting the assignment to a $B_{u}$ state.

Birnbaum and Kohler have also reported the two-photon fluorescence excitation spectrum of a dilute solution of bithiophene in crystalline $n$-hexane at $77 \mathrm{~K}^{2}$ The observed spectrum seems to have some vibrational structure. Thus the $0-0$ band is located at $36200 \mathrm{~cm}^{-1}(4.48 \mathrm{eV})$, and the vibronic band with the greatest intensity appears as a doublet (centered at 36400 and $36600 \mathrm{~cm}^{-1}$ ). It is clear that the two-photon fluorescence signal is due to a $A_{g}$ state (if $C_{2 h}$ symmetry is assumed) but the question is if it is the second or third ${ }^{1} A_{g}$ state. The authors suggest that the observed band corresponds to the $2{ }^{1} A_{g}$ state due to the close correspondence between the observed origin energy to that predicted by extrapolating the measured dithienylpolyene origin energies. The suggestion by Negri and Zgierski ${ }^{8}$ that the third $A_{g}$ is observed would require that only $S_{2}$ emission is seen in the condensed phase, which would be very surprising.

The uv absorption spectrum of bithiophene has been investigated in solution in order to provide experimental information for determining the predominant conformation of the molecule. ${ }^{29,30}$ Abu-Eittah et al. ${ }^{29}$ reported the uv absorption spectrum of bithiophene using solvents of different polarity.
Three well-defined electronic transitions were observed in the 3.75-6.20 eV energy region and assigned to $\pi \rightarrow \pi^{*}$ transitions. For the three bands, the position of the $\lambda_{\text {max. }}$ changed very little with the solvent polarity, but the relative intensity of the second and third bands was more affected by the solvent. Thus in cyclohexane the maxima of the bands appear at 4.12, 5.00, and $6.05 \mathrm{eV}(f=0.36$ and 0.22 for the two first transitions). The band at $6.05 \mathrm{eV}$ is more intense than the band at $5.00 \mathrm{eV}$, but the oscillator strength is not given. Using methanol as solvent the positions are 4.09, 5.02, and 5.93 $\mathrm{eV}$, and the oscillator strengths $0.35,0.21$, and 0.11 , respectively. Hence, in methanol the third band has less intensity than the second one. Whatever the solvent, the longwavelength band appears with the highest intensity and broad, which has been attributed to the existence of a number of nearly planar conformers of comparable energy.

A study of the polarizations of the $\pi-\pi^{*}$ transitions of biaryls, including bithiophene, was carried out by Nordén et $a l .{ }^{30}$ They reported the uv absorption spectra in cyclohexane solution and a polarized spectrum in oriented polyethylene films. The same three bands were observed but with relative intensities different from that reported by Abu-Eittah et $a .^{29}$ Thus the third band was located at $6.46 \mathrm{eV}$ in cyclohexane with the largest extinction coefficient. The longwavelength band was found to be polarized along the long axis. Mixed polarizations were observed for the second band (the angle between the transition moment and the long axis of the molecule was estimated to be $46^{\circ}$ ). The bands appeared at the same positions in solution and in film. Nordén et $a l .^{30}$ reported also PPP calculations for planar cis- and trans-bithiophene in order to elucidate the preferred conformation of the molecule by means of the comparison of the calculated and experimental spectra. A trans conformation was shown to reproduce better the experimental spectrum.

It is clear from the summary given above that the experimental situation is unclear with respect to the state ordering and the symmetry of the lowest states in bithiophene. Most evidence seems to indicate that the lowest state is of $B$ symmetry but the location of the lowest state of $A$ symmetry has not been established. Below we shall present the results of the calculations, which explain the complexity of the experimental spectrum and show that the state ordering and transition energies in this system cannot easily be related to that of the polyenes.

\section{B. A qualitative analysis}

It is tempting to try to relate the electronic states in bithiophene to those of the monomer. Such an analysis was recently, and successfully, performed for biphenyl, where it was shown that the excited states could be identified with respect to the corresponding excitations in benzene. ${ }^{31}$ However, the coupling of the two $\pi$ systems in biphenyl is rather weak (the CC bond distance is close to a single bond: 1.50 $\AA$ ). Therefore the orbitals are only weakly perturbed and retain their benzene identity to a large extent. The situation in bithiophene is different. The coupling is much stronger with a CC link distance of only $1.45 \AA$. The molecular orbitals of the dimer are therefore considerably modified, in particular, since the HOMO and HOMO-1 orbitals in thiophene are 
TABLE II. SCF $\pi$-orbital energies (in $\mathrm{eV}$ ) of thiophene and planar transbithiophene.

\begin{tabular}{ccc}
\hline \hline Thiophene $\left(C_{2 v}\right)$ & $b_{1}$ & $a_{2}$ \\
& -14.4 & -9.0 \\
& -9.5 & +5.3 \\
& +3.0 & \\
Bithiophene $\left(C_{2 h}\right)$ & $b_{g}$ & $a_{u}$ \\
& -14.0 & -15.2 \\
& -9.8 & -10.4 \\
& -7.7 & -9.6 \\
& +3.8 & +1.6 \\
& +6.0 & +5.4 \\
\hline \hline
\end{tabular}

close in energy and therefore mix strongly when perturbed (cf. Table II). It is thus not possible to identify an excitation pattern in the dimer from the orbitals of thiophene.

However, an inspection of the $\pi$-orbital energies for bithiophene shows that we can expect a complicated lowenergy spectrum involving several excited states. The HOMO to LUMO orbital energy separation is $9.3 \mathrm{eV}$, which is considerably smaller than in thiophene $(12 \mathrm{eV})$. The first ${ }^{1} B_{u}$ state is therefore expected at an energy lower than the corresponding ${ }^{1} B_{2}$ state in thiophene, which is the second excited state and appears at $5.7 \mathrm{eV}$. The HOMO- 1 energy in this molecule is only $0.5 \mathrm{eV}$ below the HOMO and it is the HOMO- $1 \rightarrow$ LUMO excitation, which gives rise to the first excited state, $2{ }^{1} A_{1}$, in thiophene at $5.3 \mathrm{eV}$. It is pushed below the ${ }^{1} B_{1}$ state due to the interaction with the $\mathrm{HOMO} \rightarrow \mathrm{LUMO}+1$ excitation (for a more detailed discussion of the thiophene spectrum see Ref. 15). It is this competition between the $\mathrm{HOMO} \rightarrow$ LUMO excitation and the interacting $\mathrm{HOMO}-1 \rightarrow$ LUMO and $\mathrm{HOMO} \rightarrow \mathrm{LUMO}+1$ configurations that determines the ordering of the lowest states in the molecules containing two interacting double bonds.

The situation in bithiophene is further complicated by the fact that the HOMO-1 and HOMO-2 orbital energies differ by only $0.2 \mathrm{eV}$. Thus four excitations, two of $A_{g}$ and two of $B_{u}$ symmetry, can be expected at energies below $5 \mathrm{eV}$. The ordering cannot be predicted from simple arguments, since it depends on the strength of the interaction between the two pairs of electron configurations. We can also expect the $(\mathrm{HOMO})^{2} \rightarrow(\mathrm{LUMO})^{2}$ double excitation will contribute strongly to the lowest $A_{g}$ states, similarly to what happens in the five-membered ring systems. It is clear that the experimental difficulties in assigning the measured spectra are related to this complexity with four states of two different symmetries within a narrow energy range.

Another possible analysis would be to compare bithiophene with cis-octatetraene, for which the $\pi$ conjugation ought to be similar. This is, however, not the case. Thiophene itself is the five-membered ring system that differs most from cis-butadiene. It is considered to be most aromatic, since the sulphur $\pi$-orbital interacts more strongly with the rest of the ring than the corresponding nitrogen or oxygen orbital in pyrrole and furan, respectively. ${ }^{15,26}$ It is therefore not possible to neglect the coupling of the sulfur atom to the carbon $\pi$ system in a discussion of the electronic structure of the polythiophenes.
The geometry of bithiophene in the ground state is trans, but slightly out of plane with a twist angle of about $38^{\circ}$. The barrier to planarity is small. ${ }^{19}$ The situation is most probably different in the excited states. The HOMO is antibonding over the dimer while the HOMO-1 and HOMO-2 orbitals are nonbonding. The LUMO is, on the other hand, bonding, while the LUMO+1 is antibonding. Thus, we expect the first ${ }^{1} B_{u}$ state $(\mathrm{HOMO} \rightarrow \mathrm{LUMO})$ to be planar. The same is most probably true for the other states, which have the LUMO orbital strongly occupied. Long vibrational progressions are expected in gas phase spectra, while spectra recorded in solid phases at low temperatures would be redshifted, if the ground state is trapped in a planar conformation.

\section{The calculated excited states and the electronic spectrum}

The six lowest singlet valence excited states have been considered, four of ${ }^{1} B_{u}$ and two of ${ }^{1} A_{g}$ symmetry $\left({ }^{1} B\right.$ and ${ }^{1} A$ in the twisted form). We shall usually use the $C_{2 h}$ labels also in this case. The main characteristics of the CASSCF wave functions are given in Table III. The first ${ }^{1} B_{u}$ state is dominated by the HOMO $\rightarrow$ LUMO excitation, but contains in addition a sizable contribution from HOMO- $\rightarrow$ LUMO. The same is true for the next ${ }^{1} B_{u}$ state even if there are now larger contributions from higher excitations.

The first state of ${ }^{1} A_{g}$ symmetry mainly corresponds to the HOMO- $1 \rightarrow$ LUMO excitation, but contains also $23 \%$ double excitations. The character of being doubly excited is even larger in the next state of this symmetry $(42 \%)$ and here it is the (HOMO $)^{2} \rightarrow(\mathrm{LUMO})^{2}$ that dominates the wave function. Thus, the characteristics of the wave functions for the four lowest excited states are those expected from the simple qualitative analysis given above.

The computed excitation energies and transition intensities for the planar and twisted forms are presented in Table IV together with relevant experimental information. The computed energy difference (CASPT2) between the two geometries in the ground state is only $0.01 \mathrm{eV}(0.25 \mathrm{kcal} / \mathrm{mol})$. A somewhat larger value $(0.81 \mathrm{kcal} / \mathrm{mol})$ was obtained in a recent MP2 calculation. ${ }^{19}$ The same calculation showed that the entire potential curve for the intramolecular rotation is very flat. The energy difference between the trans - and cisgauche forms is only $0.54 \mathrm{kcal} / \mathrm{mol}$ with a barrier of 1.56 $\mathrm{kcal} / \mathrm{mol}$ in between. This finding is confirmed by the recent measurements of the fluorescence excitation spectrum, which is shown to have two components, one which decreases with increasing temperature and one, which increases. ${ }^{4}$ The cistrans energy difference estimated from the experimental data is $1.16 \pm 0.13 \mathrm{kcal} / \mathrm{mol}$. These results are important for the present analysis for two reasons. The fact that the ground state potential is flat implies that variations in computed excitation energies for different geometries will reflect mostly the shape of the upper state potential. Second, the comparison with experiment is complicated by the fact that measured bands will depend strongly on the temperature and on the medium. It is not unlikely that the cis conformation is more stable than trans in polar media, since there is a large difference in dipole moment. It is only low-temperature gas phase data that can be directly compared to the present results. 
TABLE III. Principal configurations and weights, number and (weights) of singly, doubly, and triply excited configurations with coefficients larger than 0.05 of the ground and singlet valence excited states of planar trans-bithiophene. ${ }^{\text {a }}$

\begin{tabular}{|c|c|c|c|c|c|}
\hline \multirow[b]{2}{*}{ State } & \multirow[b]{2}{*}{ Principal configurations } & \multicolumn{4}{|c|}{ Weight } \\
\hline & & $(\%)$ & $S$ & $D$ & $T$ \\
\hline \multirow{4}{*}{$\begin{array}{l}1{ }^{1} A_{g} \\
1^{1} B_{u}\end{array}$} & $\left(3 a_{u}\right)^{2}\left(3 b_{g}\right)^{2}\left(4 a_{u}\right)^{2}\left(4 b_{g}\right)^{2}$ & 84 & & $16(9 \%)$ & \\
\hline & $\left(2 b_{g}\right)^{2}\left(3 a_{u}\right)^{2}\left(3 b_{g}\right)^{2}\left(4 a_{u}\right)^{2}\left(4 b_{g}\right)^{1}\left(5 a_{u}\right)^{1}$ & 43 & $7(78 \%)$ & $14(10 \%)$ & \\
\hline & $\left(2 b_{g}\right)^{2}\left(3 a_{u}\right)^{2}\left(3 b_{g}\right)^{1}\left(4 a_{u}\right)^{2}\left(4 b_{g}\right)^{2}\left(5 a_{u}\right)^{1}$ & 20 & & & \\
\hline & $\left(2 b_{g}\right)^{2}\left(3 a_{u}\right)^{2}\left(3 b_{g}\right)^{2}\left(4 a_{u}\right)^{2}\left(4 b_{g}\right)^{1}\left(6 a_{u}\right)^{1}$ & 7 & & & \\
\hline \multirow[t]{5}{*}{$2{ }^{1} B_{u}$} & $\left(2 b_{g}\right)^{2}\left(3 a_{u}\right)^{2}\left(3 b_{g}\right)^{2}\left(4 a_{u}\right)^{2}\left(4 b_{g}\right)^{1}\left(5 a_{u}\right)^{1}$ & 36 & $8(68 \%)$ & $15(18 \%)$ & $3(1 \%)$ \\
\hline & $\left(2 b_{g}\right)^{2}\left(3 a_{u}\right)^{2}\left(3 b_{g}\right)^{1}\left(4 a_{u}\right)^{2}\left(4 b_{g}\right)^{2}\left(5 a_{u}\right)^{1}$ & 19 & & & \\
\hline & $\left(2 b_{g}\right)^{2}\left(3 a_{u}\right)^{2}\left(3 b_{g}\right)^{2}\left(4 a_{u}\right)^{2}\left(4 b_{g}\right)^{1}\left(6 a_{u}\right)^{1}$ & 9 & & & \\
\hline & $\left(2 b_{g}\right)^{2}\left(3 a_{u}\right)^{2}\left(3 b_{g}\right)^{2}\left(4 a_{u}\right)^{1}\left(4 b_{g}\right)^{1}\left(5 a_{u}\right)^{2}$ & 6 & & & \\
\hline & $\left(2 b_{g}\right)^{2}\left(3 a_{u}\right)^{2}\left(3 b_{g}\right)^{2}\left(4 a_{u}\right)^{2}\left(5 b_{g}\right)^{1}\left(5 a_{u}\right)^{1}$ & 6 & & & \\
\hline \multirow[t]{3}{*}{$2{ }^{1} A_{g}$} & $\left(2 b_{g}\right)^{2}\left(3 a_{u}\right)^{2}\left(3 b_{g}\right)^{2}\left(4 a_{u}\right)^{1}\left(4 b_{g}\right)^{2}\left(5 a_{u}\right)^{1}$ & 50 & $7(62 \%)$ & $18(23 \%)$ & $1(<1 \%)$ \\
\hline & $\left(2 b_{g}\right)^{2}\left(3 a_{u}\right)^{2}\left(3 b_{g}\right)^{2}\left(4 a_{u}\right)^{2}\left(5 a_{u}\right)^{2}$ & 8 & & & \\
\hline & $\left(2 b_{g}\right)^{2}\left(3 a_{u}\right)^{2}\left(3 b_{g}\right)^{2}\left(4 a_{u}\right)^{2}\left(4 b_{g}\right)^{1}\left(5 b_{g}\right)^{1}$ & 6 & & & \\
\hline \multirow{4}{*}{$3{ }^{1} A_{g}$} & $\left(2 b_{g}\right)^{2}\left(3 a_{u}\right)^{2}\left(3 b_{g}\right)^{2}\left(4 a_{u}\right)^{2}\left(5 a_{u}\right)^{2}$ & 26 & $5(44 \%)$ & $19(42 \%)$ & $3(1 \%)$ \\
\hline & $\left(2 b_{g}\right)^{2}\left(3 a_{u}\right)^{2}\left(3 b_{g}\right)^{2}\left(4 a_{u}\right)^{2}\left(4 b_{g}\right)^{1}\left(5 b_{g}\right)^{1}$ & 19 & & & \\
\hline & $\left(2 b_{g}\right)^{2}\left(3 a_{u}\right)^{1}\left(3 b_{g}\right)^{2}\left(4 a_{u}\right)^{2}\left(4 b_{g}\right)^{2}\left(5 a_{u}\right)^{1}$ & 17 & & & \\
\hline & $\left(2 b_{g}\right)^{2}\left(3 a_{u}\right)^{2}\left(3 b_{g}\right)^{2}\left(4 a_{u}\right)^{1}\left(4 b_{g}\right)^{2}\left(5 a_{u}\right)^{1}$ & 5 & & & \\
\hline \multirow[t]{6}{*}{$3{ }^{1} B_{u}$} & $\left(2 b_{g}\right)^{2}\left(3 a_{u}\right)^{2}\left(3 b_{g}\right)^{1}\left(4 a_{u}\right)^{2}\left(4 b_{g}\right)^{2}\left(5 a_{u}\right)^{1}$ & 29 & $7(53 \%)$ & $18(32 \%)$ & $2(<1 \%)$ \\
\hline & $\left(2 b_{g}\right)^{1}\left(3 a_{u}\right)^{2}\left(3 b_{g}\right)^{2}\left(4 a_{u}\right)^{2}\left(4 b_{g}\right)^{2}\left(5 a_{u}\right)^{1}$ & 14 & & & \\
\hline & $\left(2 b_{g}\right)^{2}\left(3 a_{u}\right)^{2}\left(3 b_{g}\right)^{2}\left(4 a_{u}\right)^{2}\left(5 a_{u}\right)^{1}\left(5 b_{g}\right)^{1}$ & 11 & & & \\
\hline & $\left(2 b_{g}\right)^{2}\left(3 a_{u}\right)^{1}\left(3 b_{g}\right)^{2}\left(4 a_{u}\right)^{2}\left(4 b_{g}\right)^{1}\left(5 a_{u}\right)^{2}$ & 8 & & & \\
\hline & $\left(2 b_{g}\right)^{2}\left(3 a_{u}\right)^{2}\left(3 b_{g}\right)^{2}\left(4 a_{u}\right)^{2}\left(4 b_{g}\right)^{1}\left(6 a_{u}\right)^{1}$ & 8 & & & \\
\hline & $\left(2 b_{g}\right)^{2}\left(3 a_{u}\right)^{2}\left(3 b_{g}\right)^{2}\left(4 a_{u}\right)^{1}\left(4 b_{g}\right)^{1}\left(5 a_{u}\right)^{2}$ & 5 & & & \\
\hline \multirow{3}{*}{$4{ }^{1} B_{u}$} & $\left(2 b_{g}\right)^{2}\left(3 a_{u}\right)^{2}\left(3 b_{g}\right)^{2}\left(4 a_{u}\right)^{1}\left(4 b_{g}\right)^{2}\left(5 b_{g}\right)^{1}$ & 32 & $6(42 \%)$ & $26(40 \%)$ & $3(1 \%)$ \\
\hline & $\left(2 b_{g}\right)^{2}\left(3 a_{u}\right)^{1}\left(3 b_{g}\right)^{2}\left(4 a_{u}\right)^{2}\left(4 b_{g}\right)^{1}\left(5 a_{u}\right)^{2}$ & 14 & & & \\
\hline & $\left(2 b_{g}\right)^{2}\left(3 a_{u}\right)^{1}\left(3 b_{g}\right)^{1}\left(4 a_{u}\right)^{2}\left(4 b_{g}\right)^{2}\left(5 a_{u}\right)^{2}$ & 5 & & & \\
\hline
\end{tabular}

${ }^{a}$ Results from CASSCF calculations with the basis set: $C /[3 s 2 p 1 d], S /[4 s 3 p 1 d], H /[2 s]$. The orbitals $1-2 a_{u}$ and $1 b_{g}$ are not given since they appear in the list with occupation 2 everywhere.

The potential curves for the excited states are very different from those of the ground state. In the region of the trans conformer they have a distinct minimum at the planar geometry. The energy difference to the twisted geometry varies between $0.14 \mathrm{eV}$ for the $2{ }^{1} B_{u}$ state to $0.45 \mathrm{eV}$ for the $2{ }^{1} A_{g}$ state. Since the potential for the ground state is flat, these energy differences are almost identical to the difference between the two sets of excitation energies given in Table
IV. As a result, the computed excitation energies for the planar conformation are close to the $0-0$ transition energies. The computed "vertical" energies (those for the twisted form) are more difficult to relate directly to experimental data, again because the potential for the ground state is flat, while the upper potential is not. It should be noted that the transition moments vary strongly with geometry.

As expected from the qualitative analysis, we find four

TABLE IV. Calculated and experimental singlet-singlet excitation energies $(\mathrm{eV})$ and calculated oscillator strengths in planar and twisted trans-bithiophene.

\begin{tabular}{|c|c|c|c|c|c|}
\hline States & $1{ }^{1} B_{u}$ & $2{ }^{1} B_{u}$ & $2{ }^{1} A_{g}$ & $3{ }^{1} A_{g}$ & $3{ }^{1} B_{u}$ \\
\hline \multicolumn{6}{|l|}{ Planar conformation } \\
\hline CASSCF & 5.64 & 6.13 & 5.92 & 5.31 & 7.82 \\
\hline CASPT2 & 3.88 & 4.15 & 4.40 & 4.71 & 5.53 \\
\hline osc.str. & 0.062 & 0.014 & Forb. & Forb. & 0.253 \\
\hline \multicolumn{6}{|l|}{ Twisted conformation } \\
\hline CASSCF & 5.76 & 6.29 & $6.07^{\mathrm{a}}$ & 5.56 & 7.86 \\
\hline CASPT2 & 4.36 & 4.22 & $4.90^{\mathrm{a}}$ & 4.99 & 5.79 \\
\hline osc.str. & 0.049 & 0.034 & 0.013 & 0.0014 & 0.172 \\
\hline \multirow[t]{2}{*}{ Experimental energies } & \multirow{2}{*}{\multicolumn{2}{|c|}{$\begin{array}{c}3.86(0-0))^{\mathrm{b}} 3.67(0-0)^{\mathrm{c}} \\
4.13,{ }^{\mathrm{d}} 4.11,^{\mathrm{f}} 4.09^{\mathrm{g}}\end{array}$}} & \multirow{2}{*}{\multicolumn{2}{|c|}{$\begin{array}{c}4.96,{ }^{\mathrm{d}} 5.02^{\mathrm{f}, \mathrm{g}} \\
4.48(0-0)^{\mathrm{e}}\end{array}$}} & $6.46^{\mathrm{f}}$ \\
\hline & & & & & $5.93^{\mathrm{g}}$ \\
\hline
\end{tabular}

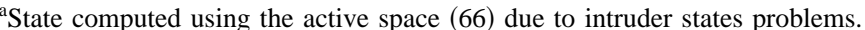

${ }^{\mathrm{b}}$ Fluorescence excitation spectrum of bithiophene seeded into a supersonic He expansion, Ref. 4.

${ }^{c}$ Fluorescence excitation spectrum of solid solutions of bithiophene in $n$-hexane at $4.2 \mathrm{~K}$ Ref. 1.

${ }^{\mathrm{d}}$ Gas-phase absorption spectrum at room temperature, Ref. 4.

${ }^{\mathrm{e}}$ Two-photon fluorescence excitation spectrum of a dilute solution of bithiophene in crystalline $n$-hexane at $77 \mathrm{~K}$, Ref. 2.

${ }^{\mathrm{f}} \mathrm{UV}$ absorption spectrum in cyclohexane, Ref. 30.

${ }^{\mathrm{g}} \mathrm{UV}$ absorption spectrum in methanol, Ref. 29. 
TABLE V. Comparison between the present excitation energies for planar bithiophene and earlier theoretical data. Energies in eV, oscillator strengths within parentheses.

\begin{tabular}{llcccc}
\hline \hline State & This work & INDO/MRDCI $^{\mathrm{a}}$ & QCPF/PI+CISD & ${\text { CNDO/S }+ \text { CISD }^{\mathrm{b}}}^{\text {PPP }^{\mathrm{d}}}$ \\
\hline $1{ }^{1} B_{u}$ & $3.88(.062)$ & 4.37 & $4.31(.76)$ & $4.25(.540)$ & $4.29(.92)$ \\
$2{ }^{1} B_{u}$ & $4.15(.014)$ & 4.72 & $5.14(.016)$ & $4.83(.025)$ & $4.96(.25)$ \\
$2{ }^{1} A_{g}$ & $4.40(-)$ & 3.91 & $4.54(-)$ & $4.25(-)$ & $5.06(-)$ \\
$3{ }^{1} A_{g}$ & $4.71(-)$ & 5.02 & $5.48(-)$ & $5.05(-)$ & $5.77(-)$ \\
$3{ }^{1} B_{u}$ & $5.53(.253)$ & - & $6.52(.635)$ & $6.04(.295)$ & $7.04(.74)$ \\
\hline \hline
\end{tabular}

${ }^{\mathrm{a}}$ From the work of Beljonne et al. using experimental geometry (Ref. 7).

${ }^{\mathrm{b}}$ Negri and Zgierski (Ref. 8).

'Pariser-Parr-Pople $\pi$-electron theory by Nordén et al. (Ref. 30).

excited states at low energies (below $5.0 \mathrm{eV}$ ). A third ${ }^{1} B_{u}$ state is found with energies around $5.5 \mathrm{eV}(0-0$ transition). The first excited state is of ${ }^{1} B_{u}$ symmetry with a computed $0-0$ energy of $3.88 \mathrm{eV}$. This is in agreement with the recent fluorescence excitation spectrum of Chadwick and Kohler, ${ }^{4}$ who place the $0-0$ transition at $3.86 \mathrm{eV}$. The corresponding spectrum in solid solution of $n$-hexane places the band at $3.67 \mathrm{eV}$, corresponding to a solvent shift of $-0.19 \mathrm{eV}$ (this is almost exactly the redshift expected if the molecule is forced to be planar in the condensed phase). The second state is also of ${ }^{1} B_{u}$ symmetry. This possibility has not been discussed earlier. The $0-0$ energy is $4.15 \mathrm{eV}$. It has considerably lower intensity in the planar geometry than the first state, but they become more similar when the molecule is twisted. Gas phase absorption spectroscopy finds the first band with a peak at $4.13 \mathrm{eV}^{4}$ The band is broad with several features on the low energy side and it is not unlikely that it contains more than one transition. Also uv absorption spectra in solution show the same band. ${ }^{29,30}$

The first band of ${ }^{1} A_{g}$ symmetry appears at $4.40 \mathrm{eV}$ in the planar conformation. It is shifted to $4.90 \mathrm{eV}$ when the molecule is twisted (notice, however, that this latter energy is computed with another active space). The two-photon excitation spectrum of Birnbaum and Kohler ${ }^{2}$ locates the $0-0$ band of a state of ${ }^{1} A_{g}$ symmetry at $4.48 \mathrm{eV}$, which is again in agreement with the present result. It is discussed, whether they see the first excited state of this symmetry or the second. The present results strongly indicates that it is the first, but the $3{ }^{1} A_{g}$ state is only $0.31 \mathrm{eV}$ higher in energy. Considering the error bars of the computed excitation energies $(0.3$ $\mathrm{eV}$ ) we cannot completely rule out the possibility that it is the third state, which is observed. The proposition of Negri et $a l^{8}{ }^{8}$ that the first excited state is of this symmetry and that for this reason the observed state is $3{ }^{1} A_{g}$ can, however, be ruled out. Both these states are forbidden for the planar geometry, but become allowed when the system is twisted. Ultraviolet absorption spectroscopy finds a second band with peak at about $5.0 \mathrm{eV}^{4,29,30}$ This band most likely contains both the ${ }^{1} A_{g}$ transitions, which in the twisted conformation are almost degenerate.

The uv absorption spectra contains a third band with maximum at about $6.0 \mathrm{eV}^{29,30}$ The calculations assign this band to the third state of ${ }^{1} B_{u}$ symmetry, which is computed to have a $0-0$ transition energy of $5.53 \mathrm{eV}$, which increases to $5.79 \mathrm{eV}$ at the twisted geometry. The uv absorption spectra have been recorded in solution and a direct comparison is therefore not possible, since the ground state conformation of the molecule is not known. It is probable that it is cis, at least in polar solvents. But the assignment of the band is clear. A fourth ${ }^{1} B_{u}$ state has actually been found with an adiabatic energy of $6.02 \mathrm{eV}$ (not included in Table IV). The band at 6 $\mathrm{eV}$ is probably composed of both these transitions. The computed intensity for the latter state is, however, very small.

Finally we should mention that the location of the lowest Rydberg state $(\mathrm{HOMO} \rightarrow 3 s)$ has also been determined. An active space of 11 orbitals was used (valence $\pi$ and one $3 s$ orbital) and the calculations were performed at the twisted geometry. The computed excitation energy was 5.36 and 5.27 $\mathrm{eV}$ at the CASSCF and CASPT2 level, respectively.

\section{Comparison with earlier theoretical results}

A number of semiempirical calculations have been performed on bithiophene and larger oligomers. It is of interest to compare with the present results, since a test of the reliability of the different semiempirical approaches can aid in evaluating the results obtained for the larger systems, where the $a b$ initio methods cannot be used. Bithiophene was studied by Nordén and co-workers in 1972 using the PariserParr-Pople $\pi$-electron model.$^{30}$ They obtained the same order of the excited states as in the present study. The computed excitation energies are somewhat larger, but the separation of the $1{ }^{1} B_{u}$ and $2{ }^{1} A_{g}$ states are similar: 0.77 vs $0.52 \mathrm{eV}$ (cf. Table V). The computed intensities are much larger than the $a b$ initio values.

Beljonne et al. used the INDO/MRCI method, which seems to be less successful than the PPP approach. ${ }^{7}$ The state ordering is different, but also this method finds four states with low excitation energies. A different choice of configurations in the $\mathrm{CI}$ expansion led to correctly ordered excitation energies. Negri and Zgierski have recently published results obtained using the CNDO/S + CISD and QCPF/PI+CISD methods. ${ }^{8}$ Also these techniques give the wrong order of states, although the latter method places the first ${ }^{1} A_{g}$ state above the first (but not the second) ${ }^{1} B_{u}$ state. These two states become degenerate with the CNDO/S method. Computed excitation energies are within $1 \mathrm{eV}$ of the present results. The general conclusion must be that the semiempirical approaches seem to be able to correctly predict the general structure of the spectrum, but fails to describe the finer details (which is not unexpected). The present results could be used to calibrate the approximate data for $2,2^{\prime}$-bithiophene. 
Extrapolation to larger oligomers could then be done using the shifts computed with a semiempirical approach. It is, however, planned to use the present approach in a similar study of $2,2^{\prime}: 5^{\prime}, 2^{\prime \prime}$-terthiophene, which will give more data for such extrapolations.

\section{CONCLUSIONS}

We have presented results from a high level $a b$ initio quantum chemical study of the electronic spectrum of bithiophene. Multiconfigurational second order perturbation theory has been used to compute the excitation energies. This method is known to produce results, which with very few exceptions, are accurate to at least $0.3 \mathrm{eV}^{26}$ We are therefore confident that the computed singlet excitation spectrum is correct. This is also born out by the comparison with experiment. The fluorescence excitation spectra of Kohler et al. give $0-0$ energies for the first two transition that are in agreement with the present results. We conclude from the calculations that four excited singlet states occur with energies below $5.0 \mathrm{eV}$, two of ${ }^{1} A_{g}$ and two of ${ }^{1} B_{u}$ symmetry. The energies of the first state of each symmetry are correctly predicted. The comparison with experiment for the second state in each of these symmetries is more difficult, since most information is available from solvent spectra at high temperature, where the conformation of the ground state is unclear. The main features of the uv absorption spectra are, however, explained and the calculated excitation energies are close to observed band maxima. We conclude that the main features of the low energy part of the electronic spectrum of bithiophene have been understood.

\section{ACKNOWLEDGMENTS}

The research reported in this communication has been supported by a grant from the Swedish Natural Science Research Council (NFR), by the Cooperación CientíficoTécnica (Ministerio de Asuntos Exteriores) of Spain, and by the DGICYT Projects No. PB91-0935 and PB91-0634. M.R. wants to thank the Conselleria de Cultura, Educació i Ciéncia de la Generalitat Valencia for a personal grant.

${ }^{1}$ D. Birnbaum and B. E. Kohler, J. Chem. Phys. 95, 4783 (1991).

${ }^{2}$ D. Birnbaum and B. E. Kohler, J. Chem. Phys. 96, 2492 (1992).

${ }^{3}$ H. Chosrovian, S. Rentsch, D. Grebner, D. U. Dahm, E. Birckner, and H. Naarmann, Synth. Met. 60, 23 (1993).
${ }^{4}$ J. E. Chadwick and B. E. Kohler, J. Phys. Chem. 98, 3631 (1994).

${ }^{5}$ D. Jones, M. Guerra, L. Favaretto, A. Modelli, M. Fabrizio, and G. Distefano. J. Phys. Chem. 94, 5761 (1990).

${ }^{6}$ M. G. Ramsey, D. Steinmüller, and F. P. Netzer, Synth. Met. 54, 209 (1993).

${ }^{7}$ D. Beljonne, Z. Shuai, and J. L. Brédas, J. Chem. Phys. 98, 8819 (1993).

${ }^{8}$ F. Negri and M. Z. Zgierski, J. Chem. Phys. 100, 2571 (1994).

${ }^{9}$ Z. G. Soos and D. S. Galvão, J. Phys. Chem. 98, 1029 (1994).

${ }^{10}$ H. Fujimoto, U. Nagashima, H. Inokuchi, K. Seki, Y. Cao, H. Nakahara, J. Nakayama, M. Hoshino, and K. Fukuda, J. Chem. Phys. 92, 4077 (1990).

${ }^{11}$ W. J. Buma, B. E. Kohler, and K. Song, J. Chem. Phys. 94, 6367 (1991).

${ }^{12}$ R. Zamboni, N. Periasamy, G. Ruani, and C. Taliani, Synth. Met. 54, 57 (1993).

${ }^{13}$ N. Periasamy, R. Danieli, G. Ruani, R. Zamboni, and C. Taliani, Phys. Rev. Lett. 68, 919 (1992).

${ }^{14}$ L. Serrano-Andrés, M. Merchán, I. Nebot-Gil, R. Lindh, and B. O. Roos, J. Chem. Phys. 98, 3151 (1993).

${ }^{15}$ L. Serrano-Andrés, M. Merchán, M. P. Fülscher, and B. O. Roos, Chem. Phys. Lett. 211, 125 (1993).

${ }^{16}$ L. Serrano-Andrés, B. O. Roos, and M. Merchán, Theor. Chim. Acta 87, 387 (1994).

${ }^{17}$ P.-O. Widmark, P.-Å. Malmqvist, and B. O. Roos, Theor. Chim. Acta 77, 291 (1990).

${ }^{18}$ P.-O. Widmark, B. J. Persson, and B. O. Roos, Theor. Chim. Acta 79, 419 (1991).

${ }^{19}$ E. Ortí, P. Viruela, J. Sánchez-Marín, and F. Tomás, J. Phys. Chem. (submitted).

${ }^{20}$ A. Almenningen, O. Bastiansen, and P. Svendsås, Acta Chem. Scand. 12, 1671 (1958).

${ }^{21}$ S. Samdal, E. J. Samuelsen, and H. V. Volden, Synth. Met. 59, 259 (1993).

${ }^{22}$ G. J. Visser, G. J. Heeres, J. Wolters, and A. Vos, Acta Cryst. B 24, 467 (1968).

${ }^{23}$ B. O. Roos, The Complete Active Space Self-Consistent Field Method and its Applications in Electronic Structure Calculations, in Advances in Chemical Physics; Ab Initio Methods in Quantum Chemistry-II, edited by K. P. Lawley (Wiley, Chichester, 1987), Chap. 69, p. 399.

${ }^{24}$ K. Andersson, P.-A. Malmqvist, B. O. Roos, A. J. Sadlej, and K. Wolinski, J. Phys. Chem. 94, 5483 (1990).

${ }^{25}$ K. Andersson, P.-Å. Malmqvist, and B. O. Roos, J. Chem. Phys. 96, 1218, (1992).

${ }^{26}$ B. O. Roos, M. P. Fülscher, P.-Å. Malmqvist, M. Merchán, and L. Serrano-Andrés, Theoretical Studies of Electronic Spectra of Organic Molecules, in Quantum Mechanical Electronic Structure Calculations with Chemical Accuracy, edited by S. R. Langhoff (Kluwer, Dordrecht, 1994).

${ }^{27}$ P. ̊. Malmqvist and B. O. Roos, Chem. Phys. Lett. 155, 189 (1989).

${ }^{28}$ K. Andersson, M. R. A. Blomberg, M. P. Fülscher, V. Kellö, R. Lindh, P.-A. Malmqvist, J. Noga, J. Olsen, B. O. Roos, A. J. Sadlej, P. E. M. Siegbahn, M. Urban, and P.-O. Widmark. Molcas Version 3. Dept. of Theor. Chem., Chem. Center, Univ. of Lund, Lund, Sweden, 1994.

${ }^{29}$ R. H. Abu-Eittah and F. A. Al-Sugeir, Bull. Chem. Soc. Jpn. 58, 2126 (1985).

${ }^{30}$ B. Nordén, R. Håkansson, and M. Sundbom, Acta Chem. Scand. 26, 429 (1972)

${ }^{31}$ M. Rubio, M. Merchán, E. Ortí, and B. O. Roos (unpublished). 